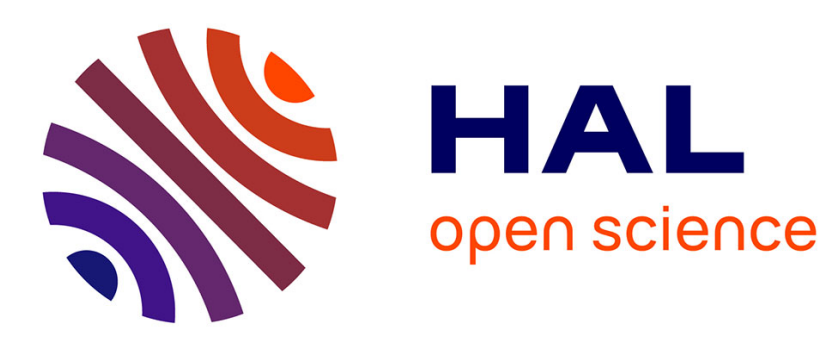

\title{
Tunable discontinuous shear thickening with magnetorheological suspensions
}

Georges Bossis, Yan Grasselli, Alain Meunier, Olga Volkova

\section{To cite this version:}

Georges Bossis, Yan Grasselli, Alain Meunier, Olga Volkova. Tunable discontinuous shear thickening with magnetorheological suspensions. Journal of Intelligent Material Systems and Structures, 2017, pp.1 - 7. 10.1177/1045389X17704915 . hal-01688293

\section{HAL Id: hal-01688293 https://hal.science/hal-01688293}

Submitted on 22 Sep 2018

HAL is a multi-disciplinary open access archive for the deposit and dissemination of scientific research documents, whether they are published or not. The documents may come from teaching and research institutions in France or abroad, or from public or private research centers.
L'archive ouverte pluridisciplinaire HAL, est destinée au dépôt et à la diffusion de documents scientifiques de niveau recherche, publiés ou non, émanant des établissements d'enseignement et de recherche français ou étrangers, des laboratoires publics ou privés. 


\title{
Tunable discontinuous shear thickening with MR suspensions
}

\author{
Georges Bossis, ${ }^{1}$ Yan Grasselli, ${ }^{2}$ Alain Meunier ${ }^{1}$ and Olga Volkova ${ }^{1}$ \\ ${ }^{1}$ Université de Nice Sophia Antipolis, Laboratoire de Physique de la Matière Condensée, CNRS UMR \\ 7336, Parc Valrose, 06108 Nice cedex 2, France \\ ${ }^{2}$ SKEMA BBA - Université de la Côte d'Azur - 60 rue Dostoievski - CS30085 - 06902 Sophia Antipolis \\ ,France
}

\begin{abstract}
Discontinuous shear thickening (DST) is a phenomena observed in concentrated suspensions where, at a given applied stress, the flow becomes suddenly partially blocked and the shear rate begins to decrease and to oscillate when the stress is increased above the critical one. In this work we show that it is possible to control with a magnetic field this abrupt transition from a flowing state to a jammed state, using a suspension of magnetic particles coated with a superplastifier molecule. In the case of experiments made at constant velocity, the transition to the jammed state corresponds to a very high jump of stress which can reach several hundred Pascal for fields as low as a few $\mathrm{kA} / \mathrm{m}$. We present also results obtained in microgravity on a magnetic powder, showing that the solid friction between particles plays a key role in the jamming phenomenon. Keywords
\end{abstract}

Discontinuous shear thickening, magnetorheology, magnetic suspensions, yield stress

\section{Introduction}

Mastering the flow of concentrated suspensions is important in numerous industrial processes like the spreading of cement or concrete, plaster, paints and so on. In most cases an important concentration of solid particles is required in order to reinforce the mechanical properties of the system after drying. The maximum packing fraction which can flow is determined by the shape and size distribution of the particles and also by the state of aggregation of the particles. Actually this last factor is quite fundamental because, even if we choose the shape and size distribution to obtain a theoretically high volume fraction, it will not be attainable if the particles begin to aggregate and to form loose clusters instead of gently pack under the effect of gravity or hydrodynamic forces. This is the reason why coating layers either made of ions or of different kinds of molecules are used to prevent the aggregation between the particles. Nevertheless even if the particles are not aggregated at low shear thanks to the coating layer, this coating can fail to prevent aggregation at higher shear and this will be the cause of a sudden shear (or stress) induced aggregation which will provoke the jamming and the DST transition. Actually this transition will happen when the applied stress will be high enough to 
overcome the repulsive force given by the coating layer so we expect that this transition will be driven by the applied stress rather than by the shear rate. On the other hand if we add to the mechanical stress an external one such as a magnetic or an electric stress, then we expect that the critical stress can be controlled externally, which is quite interesting for applications related to on/off control of a flow. In this aim we need to use either a magnetorheological or an electrorheological suspension which shows a jamming transition in the absence of field; then the application of the field will reduce the critical stress (and so the critical shear rate) at which this transition will occur. We have shown previously that some superplastifier molecules used in cement industry were able to provoke the jamming transition on suspensions made of gypsum particles or of calcium carbonate particles (Neuville et al., 2012) and very recently we have demonstrated that, using the same molecule with carbonyl iron particles, it was possible to trigger the jamming transition with the help of a magnetic field (Bossis et al., 2016). In this paper we present some new results which support the interpretation of the development of a network of frictional contacts given by Mari et al (2014) and we discuss the behavior of this network of particles under the applied stress which can be mechanical and/or magnetic. The first part is devoted to the presentation of the materials we have used and to the comparison of the jamming transition obtained with the same plastifier but two different kinds of particles: carbonyl iron and calcium carbonate. In the second part we present the effect of applying a magnetic field on the DST transition and in the third part we look to what happen above the jamming transition using for that a homemade cell. In the last part we discuss the role of frictional contacts between the particles using results obtained on a dry powder.

\section{I- Jamming transition in the presence of a superplastifier molecule.}

Most of the jamming transitions reported in the literature concern ceramic particles (Bergström L, 1998), the suspension of cornstarch particles (Fall et al, 2008), (Fall et al. 2015) or model suspensions made of PMMA (Pan et al. 2015) or silica particles (Lootens et al. 2003). More recently we have found that superplastifier molecules which are used in cement industry to increase the volume fraction of mineral particles, still keeping a low yield stress and viscosity, can also produce strong jamming transition as reported by Neuville et al (2012)). In this work we have used a polyelectrolyte molecule with a head made of two phosphonate groups $\mathrm{PO}_{3} \mathrm{H}$ connected by a Nitrogen atom : and a tail made of a polyoxyethylene chain containing $\mathrm{n}=44$ units : $\mathrm{H}-\left(\mathrm{OCH}_{2} \mathrm{CH}_{2}\right)_{\mathrm{n}}-\mathrm{N}\left(\mathrm{CH}_{2}-\mathrm{PO}_{3} \mathrm{H}_{2}\right)_{2}$ that we have 
called PPP44 and whose average molecular mass is $M_{w}=2200 \mathrm{~g} / \mathrm{mol}$. This diphosphonate was known to strongly adsorb on the surface of calcium carbonate(Mosquet et al.1997,1999). The counter-ions of the phosphonate were sodium ions.

In the presence of positive ions at the surface of mineral particles dispersed in water this molecule strongly adsorbs on the surface of the particles by ionic interaction between opposite charges. This is the case when calcium ions are present on the surface as, for example, with calcium sulfate or calcium carbonate particles but also with iron particles. In order to show this effect we have used, on one hand commercial calcium carbonate particles (BL200from Omya) and on the other hand carbonyl iron particles (grade HQ from BASF).

The average size of carbonate particles was $5.5 \mu \mathrm{m}$ and the one of carbonyl iron $1.2 \mu \mathrm{m}$, both sizes being obtained through image analysis of scanning electron microscopy pictures. The suspensions were made by dispersing the particles in distilled water with the addition of the superplastifier molecule at the concentration of $0.2 \mathrm{wt} \%$ of solid particles. This concentration was found to give the lowest viscosity and yield stress in case of calcium carbonate particles and corresponded to the coating of the surface of the particles by one molecular layer (Morini 2013) For the suspension made with carbonyl iron particles we have kept the same weight ratio and we have verified that using higher concentration of the superplastifier did not lower significantly the viscosity. The maximum volume fraction that can still flow without noticeable yield stress was $62 \%$ for carbonyl iron particles and $69 \%$ for calcium carbonate particles. The rheological measurements were made on a rheometer MCR502 from Anton Paar with a plate-plate geometry. The plates were serrated in order to prevent slippage on the wall of the plates. We also used a cover and a water trap in order to prevent evaporation of water during the experiment. We report in Fig.1 the stress versus shear rate for a ramp of stress made at a rate of $20 \mathrm{~Pa} /$ minute which is low enough to ensure that we are in the steady sate. In both cases the jamming transition is marked by the apparition of strong fluctuations of the shear rate whose average value remains approximately constant when the stress is increased and lower than the one just before the transition. The critical stresses and shear rates of iron and $\mathrm{CaCO}_{3}$ suspensions are different but they have the same order of magnitude. One can also note that the suspension of $\mathrm{CaCO}_{3}$ has a marked shear thickening behavior before the jamming transition whereas with carbonyl iron we have almost a Bingham law $: \tau=\tau_{\mathrm{y}}+\eta_{0} \dot{\gamma}$ where $\tau_{\mathrm{y}}$ is the dynamic yield stress which is here a few ten Pascal, $\eta_{0}$ the viscosity of the suspension in the absence of the field and $\dot{\gamma}$ the shear rate. 


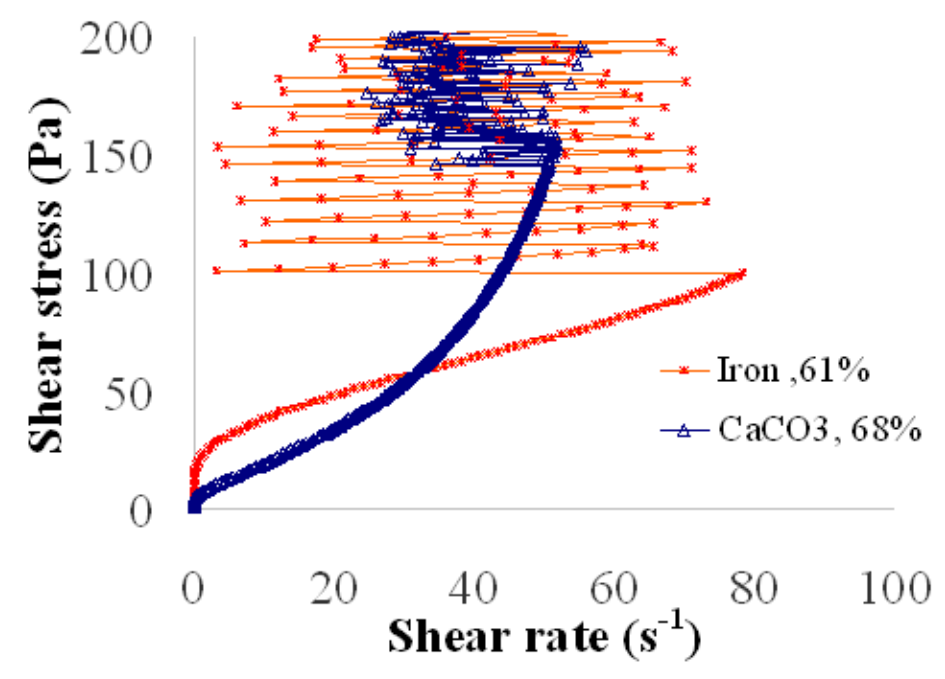

Figure 1. Jamming transition for a suspension of iron particles at $\Phi=61 \%$ and for a suspension of calcium carbonate at $\Phi=68 \%$. In both cases, the molecule PPP44 at $0.2 \%$ wt was used as adjuvant.

The critical shear stress of about $100 \mathrm{~Pa}$ was almost independent of the volume fraction contrary to the critical shear rate which decreases strongly when the volume fraction increases as can be seen in Fig.2. Also the yield stress increases with the volume fraction. Actually the molecule PPP44 is less efficient in reducing the yield stress on iron particles than on calcium carbonate ones where we can reach a volume fraction of $69 \%$ still keeping a yield stress below 5 Pascal

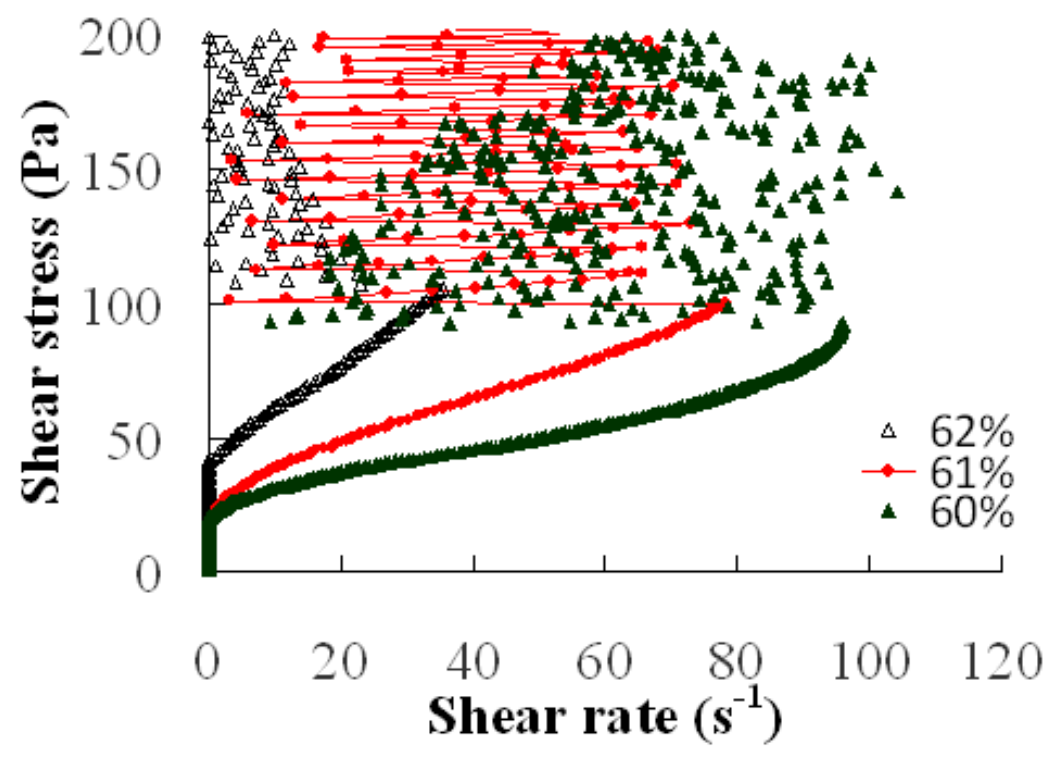

Figure 2. Evolution of the jamming transition with the volume fraction of carbonyl iron particles 
A comparison of the jamming transition at the same volume fraction for iron and $\mathrm{CaCO} 3$ particles is not possible since the jamming transition only appears above $\Phi=63 \%$ for $\mathrm{CaCO}_{3}$ suspension and the suspension of carbonyl iron does not flow at all at this volume fraction. In any event the presence of a residual yield stress at zero field does not prevent the existence of the jamming transition and consequently the possibility to control this transition with the application of a magnetic field.

\section{II-Effect of the magnetic field on the DST transition}

It is well known that usually, in magnetorheological suspensions (MRS), the stress increases with the applied magnetic field, following approximately a Bingham law :

$\sigma=\eta_{0} \dot{\gamma}+\tau_{\mathrm{m}}(\mathrm{H})$ where $\eta_{0}$ is the plastic viscosity and $\tau_{\mathrm{m}}(\mathrm{H})$ the yield stress. At low to moderate fields the yield stress increases continuously with the field and the theory of interaction between two ferromagnetic particles predicts that $\tau_{\mathrm{m}}(\mathrm{H}) \propto \Phi \mu_{0} \mathrm{M}_{\mathrm{s}}^{1 / 2} \mathrm{H}^{3 / 2}$ (Ginder et al 1996). where $\mu_{0}=4 \pi 10^{-7}$, and $M_{s}$ is the saturation magnetization (for iron $\mathrm{Ms}=1.610^{6} \mathrm{~A} / \mathrm{m}$ ). This law is quite well verified experimentally for volume fraction $\Phi<0.4$ and at low to moderate field. At higher field (B>0.3 T) Jiang et al (2015) have found that, besides the usual yield stress, a sudden increase of stress was observed at a shear rate close to $0.1 \mathrm{~s}^{-1}$ and for a volume fraction $\Phi=0.3$. Also, Ulicny et al (2010) found that adding a part of non-magnetizable particles to magnetic ones with a total volume fraction $\Phi=0.45$ could notably increase the field induced yield stress. This quite surprising observation can also be explained by a jamming phenomenon (Klingenberg, 2016). In our case and thanks to the use of the superplastifier molecule the volume fraction is much higher and, as we have seen in the previous section, the rheological curve shows a jamming transition even at zero field. We have done some experiments at imposed shear rate where we increased step by step the magnetic field. 


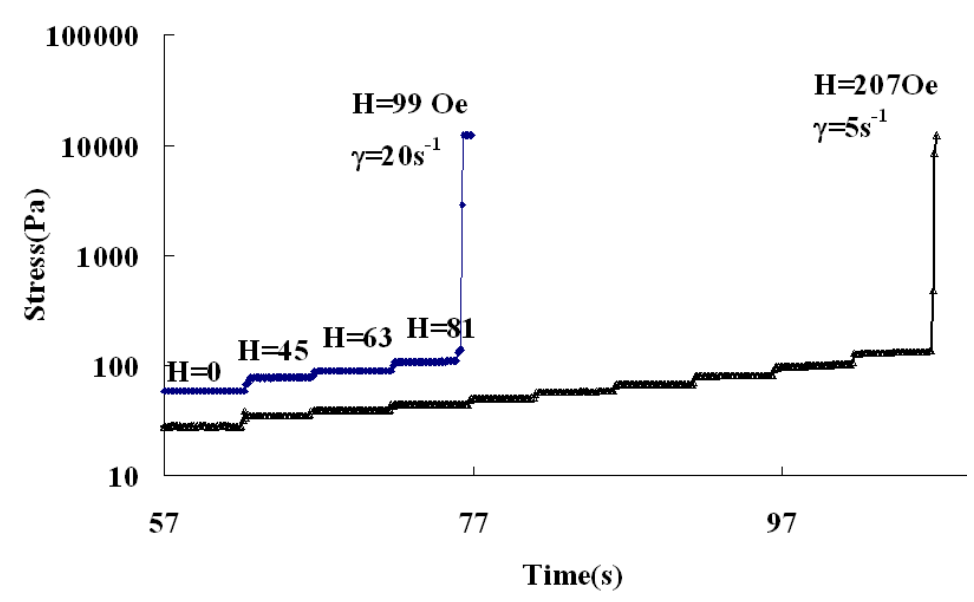

Figure 3. Evolution of the stress when the magnetic field is increased progressively with time; the shear rate is kept constant either at $20 \mathrm{~s}^{-1}$ (blue curve) or at $5 \mathrm{~s}^{-1}$ (black curve). The volume fraction is $62 \%$

The resulting stress is shown in Figure 3 for two values of the imposed shear rate: $\dot{\gamma}=20 \mathrm{~s}^{-1}$ and $\dot{\gamma}=5 \mathrm{~s}^{-1}$. The magnetic field is increased step by step with a first step from 0 to $45 \mathrm{Oe}$, and then $18 \mathrm{Oe}$ at each step. At $20 \mathrm{~s}^{-1}$ the increase of stress is quite low from $58 \mathrm{~Pa}$ at $\mathrm{H}=0$ to $76 \mathrm{~Pa}$ at $\mathrm{H}=81 \mathrm{Oe}$, then at the next increase of magnetic field we have a jump of stress to $12000 \mathrm{~Pa}$, which with our geometry -a disk of diameter $4 \mathrm{~cm}$ - corresponds to the maximum torque of the rheometer. At $5 \mathrm{~s}^{-1}$ we observe the same kind of behavior, except that the jump of stress occurs at a higher field: 189 Oe $<\mathrm{H}<207$ Oe. When the imposed shear rate is close to zero, the stress which is measured is the yield stress and this yield stress presents also a jump at a critical field $\mathrm{H}=225 \mathrm{Oe}(18 \mathrm{kA} / \mathrm{m})$ which is higher than at $5 \mathrm{~s}^{-1}$.

We see that the application of a magnetic stress contributes to decrease the critical shear rate at which the jamming occurs. This is quite understandable since the magnetic stress is an attractive one which, together with the hydrodynamic stress, will compress the particles against each other and produce the frictional contacts despite the repulsive force coming from the coating layers of PPP44. The value of the critical shear rate versus the applied magnetic field is plotted in Figure (4) for two different volume fractions of carbonyl iron: $\Phi=0.6$ and $\Phi=0.62$. 


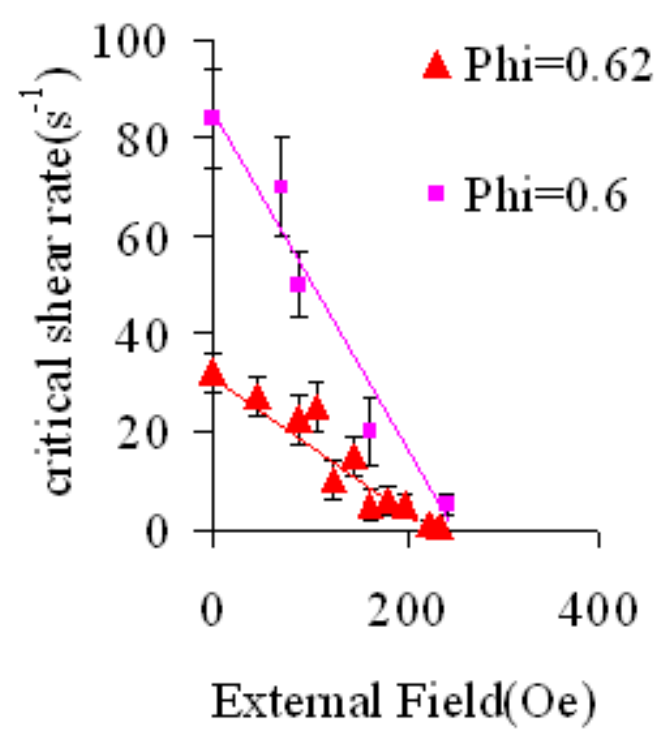

Figure 4. Evolution of the critical shear rate with the field for two volume fractions of carbonyl iron $\Phi=0.6$ and $\Phi=0.62$

The decrease of the critical shear rate is approximately linear with the field with a slope which increases when the volume fraction decreases. These lines represent the boundary between a classical MR fluid which can flow if the stress is above the yield stress -this is the domain below the boundary- and a domain above this boundary where the suspension is jammed. The graph of Figure 4 was obtained by setting the shear rate at a constant value and increasing the field until we obtained the jump of stress. It can also be obtained by doing a ramp of stress at constant field and in this case we record the critical shear rate which is the one above which we have a decrease of shear rate and an oscillation regime as shown in Fig.2. This oscillation regime above the jamming point was also found with the calcium carbonate particles. Actually if, instead of a ramp of stress, we apply a constant stress and record the shear rate versus the time we observe a regime of regular oscillations as shown in Figure.5

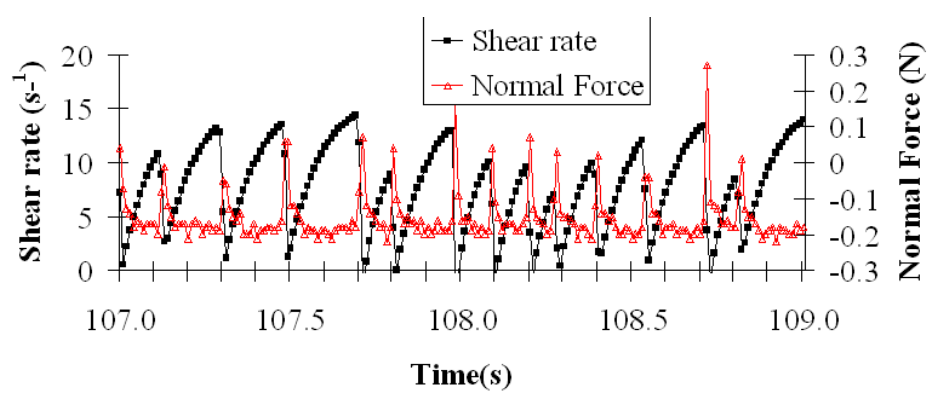

Figure 5. Regime of oscillation of the shear rate for a constant stress $\sigma=120 \mathrm{~Pa}$ and a constant field $\mathrm{H}=117 \mathrm{Oe}$. The red curve is the normal force reported on the left axis 
These oscillations are characteristics of a kind of stick-slip behavior with an asymmetric shape made of a progressive increase of shear rate followed by an abrupt decrease during the jamming phase. It can even present a negative part meaning that the upper plate is rotating back a short moment after the jamming; this quite surprising observation is very likely related to a coupling between the inertia of the tool and the elasticity of the fluid which appears above the jamming point. The triangles in Fig.5 represent the evolution of the normal force, which is constant and slightly negative except during a short time of 0.01 to $0.02 \mathrm{~s}$ where we have a positive peak of normal force. A possible interpretation is the following: in the jamming domain, the stress is high enough to induce a transient aggregation on the compression axis so the percolating network of the frictional contacts is formed progressively (the period of the oscillations corresponds to a strain of a few unities) and reaches the size of the cell. At this point the systems is jammed and the shear rate begins to decrease, but at the same time, the inertia moment of the tool is transferred as a supplementary stress on the suspension which will still contribute to push the particles against each other and to sweep the polymer out of the surface until they come in solid contact. When the particles are stopped, the lubrication flow between the surfaces no longer exists, so the polymer can come back inside the contact zone and separate again the surfaces, hence the progressive rupture of the contacts and the beginning of a new cycle. The peak in the normal force is obviously related to the sudden formation of the percolated network when the shear force is large enough to repel the polymer. The fact that it immediately drops indicates that this percolation induces a rearrangement of the particles which break in a few places the percolated network. The interplay between the percolation of a frictional network of contacts and its desagregation due to the reversibility of the adsorption of the polymer at low shear can then explain qualitatively these oscillations.

When we still increase the stress the suspension ends up to be gradually expelled from the gap between the disks. Actually it is not due to inertia forces but to the radial stress which is developed when the network of particles in contact is formed; the particles are pushed outside the liquid when this radial stress becomes larger than the capillary pressure: $p=\gamma / \mathrm{r}$ where $\gamma$ is the surface tension of water: $73 \mathrm{mN} / \mathrm{m}$ and $\mathrm{r}$ the radius of the particles or of an aggregate of particles (Cates et al, 2005). It happens in any open geometry and the only way to prevent the progressive exit of the particles and the foaming at the surface is to use a sealed cell at the price of a residual friction on the O'ring. 


\section{Rheological behavior at high stress in the jammed domain}

We have designed a new rheometer with a cell which is sealed by a Teflon ring in order to prevent the expulsion of the liquid at high stress. The velocity is controlled by a stepper motor and the torque is measured by strain gauges mounted on the rotating axis, this is a commercial rheometer developed by CAD Company, mainly for the rheology of concrete. The maximum torque is $10 \mathrm{~N} . \mathrm{m}$ that is to say 30 times more than usual rheometers. The concentric rotation of the tool is ensured by a double ball bearing. In order to prevent the suspension to slip we have used for the rotating part either a vane model or a double helicoidal ribbon (.Aït-Kadi et al, 2002)

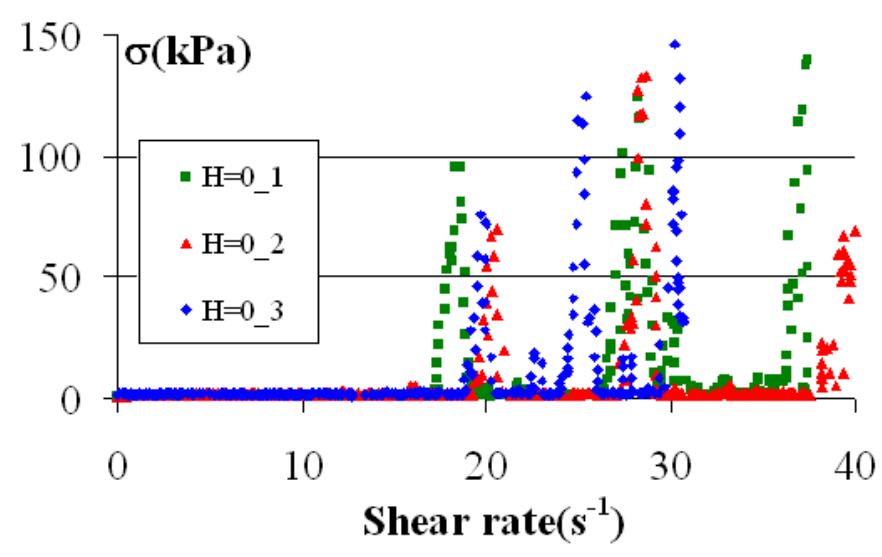

Figure 6. Suspension of carbonyl iron in water at $\Phi=62 \%$ with PPP44 as adjuvant. Three successive ramps of shear rate on the same sample at zero field

The external cylindrical cage has regular stripes of depth $0.2 \mathrm{~mm}$ also to prevent the slipping on the walls. The external diameter of the tools are both of $24 \mathrm{~mm}$ and the gap is $2 \mathrm{~mm}$. With this rheometer we can explore much higher stress: up to $150 \mathrm{kPa}$ with our cell (a mechanical safe guard limits the torque at about 8 N.m). In Figure 6 we have reported the stress obtained at zero field during a ramp of shear rate for three successive experiments. We see that the first jamming transition is quite reproducible and occurs at a shear rate between 18 and $20 \mathrm{~s}^{-1}$. After a maximum stress between 75 and $100 \mathrm{kPa}$, the stress comes back to zero and then increases again at a still higher value when the shear rate is increased. In this regime, even at constant shear rate above the critical one, we have oscillations of the stress from very high values to zero, meaning that the network of frictional contacts can break at high enough stress but will reform later on. Now, if we apply the magnetic field, which is parallel to the axis of rotation of the tool, the jamming behavior is quite different. For instance we have plotted in Figure 7 the results obtained in the presence of a magnetic field. A ramp of shear rate was 
applied at $\mathrm{t}=0$ and the maximum shear rate, here $10 \mathrm{~s}^{-1}$, was reached after $7 \mathrm{~s}$. The upper curve was obtained with the helicoidal tool and the lower one with the vane tool on the same suspension of carbonyl iron in water at a volume fraction of $61 \%$ and with $0.2 \%$ in weight of PPP44 relatively to the mass of iron. The first important observation is that, contrary to what happen at zero field, the jammed state persists whereas the suspension continues to be sheared at the shear rate of $10 \mathrm{~s}^{-1}$, resulting in a high stress (around $150 \mathrm{kPa}$ ) which is maintained despite the low value of the magnetic field. This is to be compared to the maximum yield stress of MR fluids which is usually around $50 \mathrm{kPa}$ but for much higher fields of approximately 50000 e.

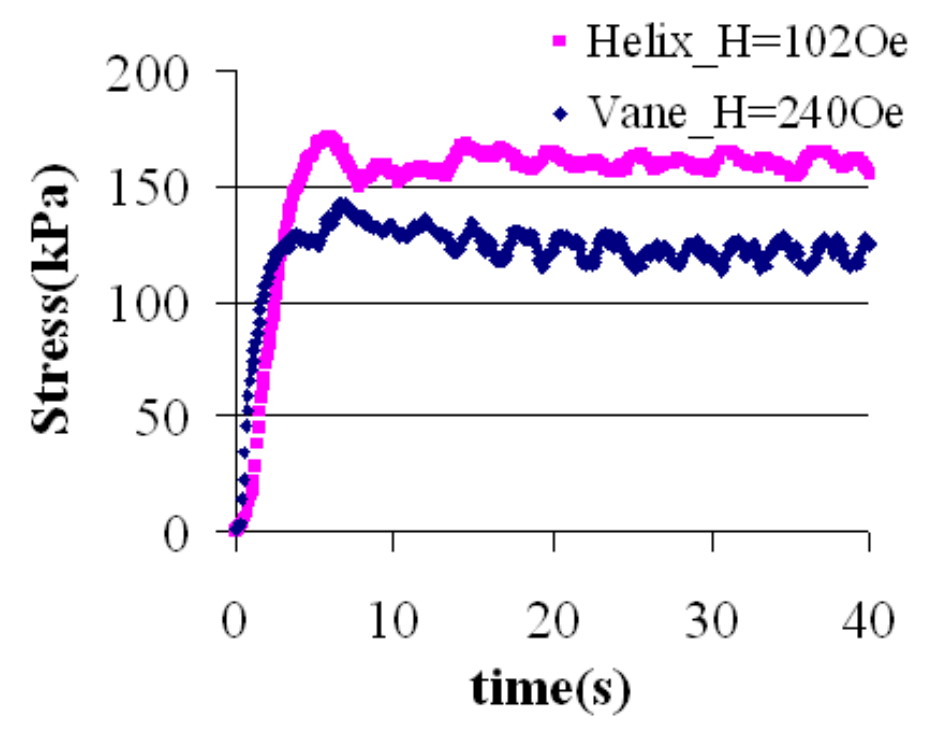

Figure 7. Stress versus time for carbonyl iron suspension $\Phi=61 \%$. Upper curve: double helix geometry; lower curve: vane geometry. Ramp of shear rate from 0 to $10 \mathrm{~s}^{-1}$ in $7 \mathrm{~s}$. The field is applied before starting the ramp.

The second point is that the helicoidal tool gives a higher stress for a field which is two times less than with the vane tool. Actually the vane tool is equivalent to a cylindrical one made of the suspension since the part inserted between the blades is driven in the same way as if it was a solid. On the other hand the helicoidal tool gives a more complex flow, since it pushes the suspension along the axis of rotation producing a backflow in the gap which superposes to the shear flow. It is possible that the jamming of this secondary flow is easier to obtain and then contributes to lower the critical field. The stress of $150 \mathrm{kPa}$ is not at all the highest one that we can obtain, but we are not able to measure it at higher field due to the torque limitation on the motor. 


\section{IV discussion of the mechanism of jamming}

We have seen the very important role of the superplastifier molecule since it allows to increase the volume fraction of carbonyl iron up to $62 \%$ by preventing the particles to aggregate in the presence of the Van Der Waals forces. If we try to densify the carbonyl iron powder, by tapping the bottom of the container, we shall reach at most a volume fraction of $42-43 \%$ with the same particles. Nevertheless it is interesting to look for the rheological behavior of this powder. This is not possible on a conventional rheometer since, due to frictional contacts, the suspension dilates if we use a cell with a free boundary. This is not the case with our sealed cell, so we have made some experiments to characterize the rheological behavior of the powder. As sedimentation can also contribute to skew the results we took advantage of parabolic flight offered by the CNES to check the effect of the sedimentation. The duration of a parabola is 22 seconds; the field is turned on at the beginning of the parabola and a constant shear rate is set $7 \mathrm{~s}$ before the microgravity period at a value of $11 \mathrm{~s}^{-1}$.

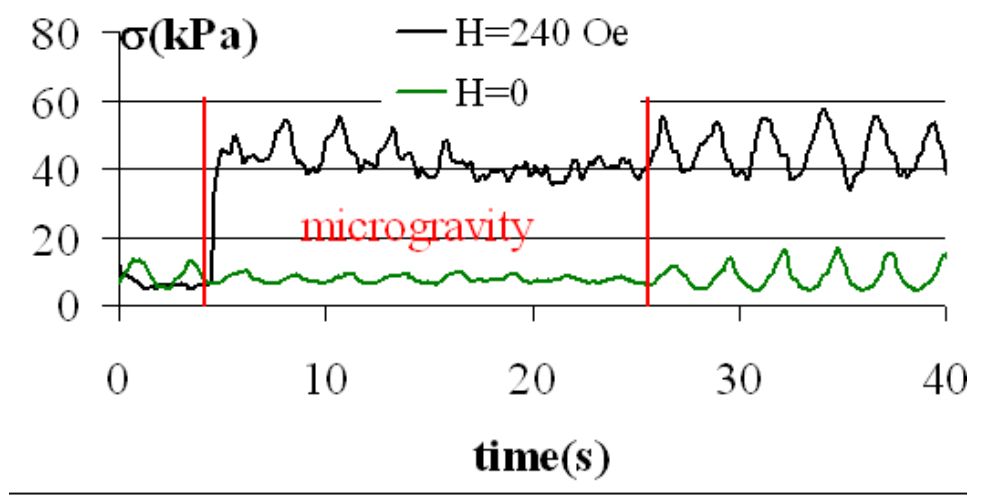

Figure 8. Stress versus time at a constant shear rate of $11 \mathrm{~s}^{-1}$ with the vane geometry in microgravity experiments upper curve: $\mathrm{H}=240$ Oe; lower curve $\mathrm{H}=0$.

The result is shown in Figure 8 at zero field and at a field $\mathrm{H}=240 \mathrm{Oe}$ which is turned on at the beginning of the microgravity period. We note that, in the absence of field, the stress is already quite high: $8 \mathrm{kPa}$ and that we have a strong oscillation of amplitude $\sim 7 \mathrm{kPa}$ which almost disappears during the microgravity period. The period of this oscillation corresponds to the one of the rotation of the tool and comes likely from a lateral oscillation of the tool provoked by a slight difference of density between the upper and lower part of the horizontal cell. In granular media the stress is proportional to the square of the shear rate and to the square of the diameter of the particles (Bagnold's law). With this volume fraction, in the 
collisional regime it should be a stress smaller than $1 \mathrm{~Pa}$ (Bossis et al, 2004) but instead we find a stress of $8 \mathrm{kPa}$ and which does not depend significantly of the shear rate. This indicates that the measured stress is not a kinetic one induced by the collision between particles but comes from the solid friction between particles which form transient networks of solid contacts between the two walls of the cell. In other words, due to the small diameter of the particles, we are in a quasi-static regime with the Savage number $S=\frac{\rho_{\mathrm{p}} \mathrm{d}^{2} \dot{\gamma}^{2}}{\tau}$ less than $10^{-7}$ (Lu et al, 2007) where $\rho_{p}$ is the density of the particles and $d$ their diameter. In this situation we see that the magnetic field is very efficient to mobilize the friction between the particles since we have an increase of $32 \mathrm{kPa}$ for a field of $240 \mathrm{Oe}$. It is interesting to compare this increase of frictional stress due to the application of the magnetic field to the one which would be obtained in the absence of solid friction between the particles. In this last case the magnetic stress is the yield stress of a suspension at a volume fraction of $42 \%$ and for a field of 2400e. Using the model of Ginder (1996), which well applies to particles of high permeability at low field, we have: $\tau_{\mathrm{y}}=\sqrt{6} \Phi \mu_{0} \mathrm{M}_{\mathrm{s}}^{1 / 2} \mathrm{H}^{3 / 2}$ where $\mathrm{M}_{\mathrm{s}}$ is the saturation magnetization and $\mu_{0}=4 \pi 10^{-7}$. Taking $\mu_{0} \mathrm{M}_{\mathrm{s}}=2 \mathrm{~T}$ for the saturation magnetization of iron we obtain $\tau_{\mathrm{y}}=403 \mathrm{~Pa}$ for $\mathrm{H}=240 \mathrm{Oe}$, this is about two orders of magnitude less than the observed increase of stress. It then appears that the frictional stress resulting from contact forces when the suspension is sheared at constant volume fraction is amplified by the application of the magnetic stress and the total stress is much larger than the addition of the frictional stress and of the magnetic stress. This amplification can be thought to be a kind of ratcheting effect where the attractive magnetic field contributes to block the network of particles in surface's asperities, preventing them to slip on each other. We can infer from this experiment on the powder that the same mechanism is acting on the wet suspension, but it is amplified due to the higher volume fraction obtained thanks to the use of superpastifier molecules. A better understanding of the jamming process in the presence of superplastifier molecules should require the measurement of repulsion forces between polymer layers adsorbed on particles, as already done with AFM (Taunton et al, 1988), (Klein et al 1994) in order to get a better information on the force needed to sweep the polymers out of the contact zone.

\section{Acknowledgments}

The authors want to thank the CENTRE NATIONAL D'ETUDES SPATIALES (CNES, the French Space Agency) for having supported this research and the company CAD for his help in developing the high torque rheometer. 


\section{References}

Aït-Kadi A., Marchal P , Choplin L, Chrissemant A.S.and Bousmina M(2002) Quantitative analysis of Mixer-type rheometers using the Couette analogy. The Canadian Journal of Chemical Engineering, 80(6): 1166-1174

Bergström L (1998) Shear Thinning and shear thickening of concentrated ceramic suspensions.

Colloids and Surfaces A: Physicochemical and Engineering Aspects 133,151155

Bossis G, Grasselli Y,and Volkova O (2004) Granular rheology in zero gravity. Journal of physics: Condensed Matter 16 (18) 3279-3287

Bossis G, Grasselli Y,Meunier A and Volkova O (2016) Outstanding magnetorheological effect based on discontinuous shear thickening in the presence of a superplastifier molecule. Applied Physics Letters 109, 111902 (2016)

Cates ME, Haw MD and Holmes CB (2005) Dilatancy,jamming and the physics of granulation Journal of Physics: Condensed Matter 17, S2517

Fall A, Huang N, Bertrand F, Ovarlez G, and Bonn D(2008) Shear Thickening of Cornstarch Suspensions as reentrant jamming transition. Physical Review Letters 100, 018301

Fall A., Bertrand F, Hautemayou D, Mezière C, Moucheront P, Lemaître A, and Ovarlez G (2015) Macroscopic Discontinuous Shear Thickening versus Local Shear Jamming in Cornstarch. Physical Review Letters 114, 098301

Ginder JM, Davis L.C and Elie LD (1996) Rheology of magnetorheological fluids: models and measurements. International Journal of Modern Physics B 10, 3293

Jiang J, Hu G., Zhang Z, Meng Y and Tian Y (2015) Stick-slip behavior of magnetorheological fluids in simple linear shearing mode. Rheologica Acta 54(9-10): 859867

Klein J., Kumachava E, Mahalu D, Perahia D, and Fetters LJ(1994) Reduction of frictional forces between solid surfaces bearing polymer brushes. Nature materials 370, 634-636

.Klingenberg DJ (2016) A Jamming-Like Mechanism of Yield Stress Enhancement in MR Suspension Mixtures of Magnetizable and Nonmagnetizable Particles communication at ERMR2016, July 4-8, 2016 Incheon, Korea

Lootens D, Van Damme H, and Hébraud P(2003) Giant Stress Fluctuations at the Jamming Transition . Physical Review Letters 90,178301

Lu K, Brodsky E and Kavehpour H(2007) Shear-weakening of the transitional regime for granular flow: the role of compressibility. Journal of Fluid Mechanics 587, 347-372

.Mari R, Seto R, Morris JF, and Denn MM(2014) Shear thickening, frictionless and frictional rheologies in non-Brownian suspensions. J. Rheol. 58, 1693-1724 (2014) 
Morini R, Bossis G, Boustingorry P and Persello J (2013) Adsorption de polyélectrolytes en relation avec la rhéologie d'une suspension de carbonate de calcium., In: Proceedings du congrès français de mécanique, CFM2013, 26-28 August 2013, Bordeaux, France 